\title{
Th2 responses induced by epicutaneous or inhalational protein exposure are differentially dependent on IL-4
}

\author{
Christina A. Herrick, ${ }^{1,2}$ Heather MacLeod, ${ }^{2}$ Earl Glusac,, ${ }^{1,3}$ Robert E. Tigelaar,, ${ }^{1,2}$ \\ and Kim Bottomly,2 \\ ${ }^{1}$ Department of Dermatology, \\ ${ }^{2}$ Section of Immunobiology, and \\ ${ }^{3}$ Department of Pathology, Yale University School of Medicine, New Haven, Connecticut 06520, USA \\ Address correspondence to: Christina A. Herrick, Department of Dermatology, Yale University School of Medicine, \\ 333 Cedar Street, PO Box 208059, New Haven, Connecticut 06520-8059, USA. Phone: (203) 737-1210; \\ Fax: (203) 785-7637; E-mail: christina.herrick@yale.edu.
}

Received for publication October 6, 1999, and accepted in revised form February 8, 2000.

\begin{abstract}
Atopic individuals are predisposed to mounting vigorous Th2-type immune responses to environmental allergens. To determine the factors responsible, animal models that closely mimic natural modes of allergen exposure should prove most informative. Therefore, we investigated the role of IL-4, a known Th2-promoting cytokine, in generation of Th2 responses after exposure of either the skin or airway to soluble protein. Compared with wild-type (WT) mice, IL-4-deficient $\left(I L-4^{-/-}\right)$mice showed markedly impaired Th2 activation after primary exposure to inhaled ovalbumin (OVA), with decreased OVA-specific IgG1 and IgE, and significantly fewer eosinophils in bronchoalveolar lavage (BAL) fluid after airway challenge. In contrast, $I_{-4^{-/}}$mice initially exposed to epicutaneous (e.c.) OVA mounted Th2 responses equivalent to responses in WT mice, with high numbers of eosinophils in BAL fluid. Because Th2 responses were not induced by e.c. OVA exposure in Stat $6^{-/}$mice (mice lacking signal transducer and activator of transcription 6), the role of IL-13 was tested. In vivo depletion of IL-13 prevented Th2 responses induced by e.c. OVA exposure in $I L-4^{-/-}$mice. These data demonstrate a marked difference in the IL-4 dependence of Th2 responses generated at two anatomic sites of natural allergen encounter and identify the skin as a particularly potent site for Th2 sensitization.
\end{abstract}

J. Clin. Invest. 105:765-775 (2000).

\section{Introduction}

The cardinal feature of atopic disease, whether allergic rhinitis, atopic dermatitis, or allergic asthma, is exuberant CD4 Th2-cell activation. This is evidenced by the predominance of Th2 cytokines such as IL-4, IL-5, and IL-13 at sites of acute allergic inflammation, including lesional skin of atopic dermatitis patients and airways of allergic asthmatics (1-6). IL-4 is a major inducer of IgE class switching $(7,8)$, accounting for the elevated levels of both total and allergen-specific IgE found in serum of atopic individuals compared with the general population. IL-5 is known to promote the differentiation, maturation, and endothelial adherence of eosinophils (9), which are found in high numbers at sites of allergic inflammation. What remains unclear is why atopic individuals so readily mount these Th2 responses to commonly encountered environmental antigens, when the majority of the population does not. Determination of the factors responsible for this ready state of Th2 activation in atopic individuals should aid in directing new therapeutic or ultimately, preventative, strategies.

During the initiation of an immune response, factors purported to skew differentiation of naive CD4 T cells toward either Th1 or Th2 effector phenotypes include the dose of antigen, strength of T-cell receptor ligation, and the cytokine microenvironment (10-13). In particular, IL-4 has been identified as a critical Th2 differentiation factor $(14,15)$. An important question, therefore, is whether or not IL-4 plays a major role in the natural course of allergen-induced Th2 activation in atopic individuals. Different mouse models have yielded conflicting data with regard to the need for IL-4 to generate Th2 responses in vivo (16-18). Numerous studies have demonstrated either a complete loss or marked reduction of Th2 responses in IL-4-deficient $\left(\mathrm{IL}^{-4^{-/}}\right)$ mice, particularly in response to protein antigens and in models of allergic airway inflammation (16, 19-23). However, IL-4-independent Th2 responses have also been demonstrated in models involving live pathogens, including Leishmania (18), Schistosoma (24), and Plasmodium (25) infections. In addition, several groups have shown that $I L-4^{-/-}$mice infected with the nematode Nippostrongylus brasiliensis are able to effectively expel adult worms from the gut - a process known to be dependent on Th2 activation - and that this IL-4-independent Th2 response is mediated by IL-13 (26-29).

Taken together, data from the literature suggest that the role of IL-4 in generation of Th2 responses in vivo may differ depending on whether the initiating stimulus is a protein antigen or a live pathogen. As stated above, IL-4-independent Th2 generation has been 
described after infection with live pathogens, but studies involving immunization with protein antigen have indicated a strong reliance on IL-4 for Th2 activation. Additional factors, such as initial route of exposure to foreign antigen or pathogen may also account for differences in the IL-4 dependence of Th2 responses in these models. More information is needed to clarify the extent to which IL-4 plays a role in the type of Th2 responses generated by atopic individuals in response to environmental allergen exposure.

To determine the contribution of IL-4 to the preferential Th2 activation demonstrated by atopic individuals, we investigated the role of IL- 4 in Th2 responses generated at anatomic sites where environmental allergens are normally encountered, namely the airway and skin. We have established sensitization protocols that result in strong Th2 activation involving initial exposure of mice to soluble ovalbumin (OVA), either by inhalation or epicutaneous (e.c.) application under an occlusive skin patch. Using $I L-4^{-/-}$mice, we show that Th2 responses to inhaled (i.n.) OVA are diminished in the absence of IL-4, whereas Th2 responses after e.c. exposure to OVA remain intact. However, epicutaneously induced Th2 responses were not seen when Stat $6^{-/}$mice (which are deficient in signal transducer and activator of transcription 6) were exposed to OVA. Instead, these mice mounted Th1-type responses. Thus, e.c. OVA exposure could induce Th2 activation independently of IL-4, but not independently of STAT6, suggesting that IL-13, another known activator of the STAT6 signaling pathway, might be involved. In vivo IL-13 depletion of epicutaneously sensitized $I L^{-4^{-/-}}$mice led to a loss of Th2 responses, confirming this hypothesis. These data implicate the skin of atopic persons as a potentially important site of initial Th2 sensitization to environmental allergens, perhaps allowing sensitization to occur even more readily than through the airway.

\section{Methods}

Animals. C57BL/6J mice were purchased from The Jackson Laboratory (Bar Harbor, Maine, USA). IL-4-/mice (C57BL/6J-I14tm2Nnt) were obtained from The Jackson Laboratory and then subsequently bred in our facilities. Stat $6^{-1-}$ mice on a B6/129 background were provided by Dr. James Ihle (St. Jude's Research Center, Memphis, Tennessee, USA), and were bred and maintained in our facilities. Female mice, $8-12$ weeks of age, were used in all experiments.

Sensitization protocols. For e.c. sensitization, mice had their backs shaved with electric clippers. One to 2 days later (day 0), $100 \mu \mathrm{L}$ of either OVA (Grade V; Sigma Chemical Co., St. Louis, Missouri, USA) $(1.0 \mathrm{mg} / \mathrm{mL})$ in PBS or PBS alone was applied to gauze in the center of an occlusive patch (DuoDERM Extra Thin; ConvaTec, Princeton, New Jersey, USA), which was then affixed to their backs. Patches were left intact for 4 days, with application of a second patch on day 14 in some cases. For i.n. sensitization, mice were anesthetized lightly with methoxyflurane (Metofane; Mallinckrodt Veterinary Inc. Mundelein, Illinois, USA) and then administered $50 \mu \mathrm{L}$ of either OVA $(2 \mathrm{mg} / \mathrm{mL})$ in PBS or PBS alone by intranasal droplet application on days 0,1 , and 2 .

Airway challenge. Beginning on day 14 after e.c. or i.n. sensitization, mice were challenged with i.n. OVA over a 6-day period. Mice were anesthetized with methoxyflurane and then administered $50 \mu \mathrm{L}$ of OVA in PBS $(0.5$ $\mathrm{mg} / \mathrm{mL}$ ) by intranasal droplet on days $14,15,18$, and 19 . Mice were sacrificed on day 21 for assessment of lung inflammatory responses.

In vivo IL-13 depletion. To block IL-13 activity in vivo, a soluble IL-13 receptor $\alpha 2-\mathrm{Fc}$ fusion protein (IL$13 \mathrm{R} \alpha-\mathrm{Fc})$, kindly provided by Genetics Institute Inc. (Cambridge, Massachusetts, USA) was used. IL-13R $\alpha$ Fc and control human IgG (ctrl-Fc) were prepared as described previously (30). $I L-4^{-/}$mice were injected intraperitoneally with $200 \mu \mathrm{g}$ of either IL-13R $\alpha$-Fc or ctrl-Fc in $500 \mu \mathrm{L}$ of PBS every other day, from day -1 to day 19 of the e.c. sensitization and airway challenge protocols described above.

Analysis of bronchoalveolar lavage (BAL) fluid. After mice were sacrificed by $\mathrm{CO}_{2}$ asphyxiation, BAL was performed by cannulation of the trachea, followed by lavage of airway lumina 3 times with $1 \mathrm{~mL}$ of PBS. After cells were washed and counted, cytospin preparations were stained with Diff-Quik (Dade Behring Inc., Newark, Delaware, USA). Differential cell counts were based on morphology and staining characteristics of 200 cells per sample.

Lung histology. Lungs were prepared by perfusion via the right ventricle with $20 \mathrm{~mL}$ of PBS and inflation with $1.0 \mathrm{~mL}$ of formalin. Paraffin-embedded lungs were sectioned in the coronal plane, ensuring that central airways were visible. Sections ( $5 \mu \mathrm{m}$ thick) were stained with hematoxylin and eosin (H\&E) or periodic acid-Schiff (PAS). A histologic inflammation index was determined by scoring H\&E-stained sections for amount and density of perivascular, peribronchiolar, and when present, interstitial infiltrate in a blinded fashion on a subjective 12 -point scale $(1=$ minimal to absent infiltrate; 12 = florid infiltrate, diffusely involving the peribronchiolar regions and pulmonary interstitium). Lungs from individual mice were scored twice by a single investigator, and the average score was used as the index value for that animal. Data are reported as mean \pm SEM of the index value.

Isolation of lung inflammatory cells. In some experiments, cells were isolated from lung tissue on day 21 by enzymatic digestion. Lungs from several mice were pooled, manually minced into fragments (1-2 mm), and incubated at $37^{\circ} \mathrm{C}$ for 20 minutes in a digestion mixture consisting of Click's medium (GIBCO BRL, Grand Island, New York, USA), 10\% FCS, 150 U/mL collagenase (Worthington Biochemical Corporation, Freehold, New Jersey, USA), and $20 \mu \mathrm{g} / \mathrm{mL}$ DNase I Type IV from bovine pancreas (Sigma Chemical Co.). After digestion, tissue was pushed through a metal strainer, filtered through nylon mesh, and centrifuged 
over a Ficoll gradient (LSM; ICN Biomedicals Inc., Aurora, Ohio, USA) to isolate mononuclear cells.

Cytokine production by lung cells. Syngeneic $\mathrm{T}$ cell-depleted splenocytes were used as antigen-presenting cells and were prepared by antibody-mediated complement lysis, using antibodies to CD4 (GK1.5), CD8 (TIB 105), and Thy1 (Y19), followed by treatment with mitomycin C. Lung cells $\left(2 \times 10^{6} / \mathrm{mL}\right)$ were cultured with antigen-presenting cells $(2 \times$ $\left.10^{6} / \mathrm{mL}\right)$ and OVA $(100 \mu \mathrm{g} / \mathrm{mL})$ in Click's medium with $5 \% \mathrm{FCS}$ at $37^{\circ} \mathrm{C}$ for 48 hours. Cytokines in culture supernatants were detected using commercially available ELISA kits (IFN- $\gamma$, IL-4, IL-5, or IL-13). The IL-13 ELISA kit was purchased from R\&D Systems (Minneapolis, Minnesota, USA); all others were from Endogen Inc. (Woburn, Massachusetts, USA). The lower limit of sensitivity for each ELISA was 0.6 $\mathrm{ng} / \mathrm{mL}$ (IFN- $\gamma$ ), $18 \mathrm{pg} / \mathrm{mL}$ (IL-4), $63 \mathrm{pg} / \mathrm{mL}$ (IL-5), and $6 \mathrm{pg} / \mathrm{mL}$ (IL-13).

Flow cytometry. Cells were preincubated with rat antimouse Fc receptor antibody (24G2) before staining with Quantum Red-conjugated anti-CD4 (clone H129.19; Sigma Chemical Co.) and PE-conjugated anti-CD8 (Ly3.2; 53-5.8; PharMingen, San Diego, California, USA).

Antibody measurement by ELISA. For most experiments, serum was collected on day 13 after e.c. or i.n. sensitization, and again at the time of sacrifice on day 21 . For studies in which mice received 2 e.c. OVA exposures, serum was obtained on days 13 and 27.

For determination of OVA-specific antibodies, 96well microtiter plates (Nalge Nunc International, Rochester, New York, USA) were coated with OVA (50 $\mu \mathrm{g} / \mathrm{mL}$; Sigma Chemical Co.) in $0.1 \mathrm{M} \mathrm{NaHCO}_{3}$ at $4^{\circ} \mathrm{C}$ overnight. The following steps were performed, with plates being washed 3 times with PBS/0.05\% Tween-20 between each step. Blocking was performed with $1 \%$ BSA in borate saline (BSA-BS) for 1 hour at $37^{\circ} \mathrm{C}$, and then sera were diluted serially in $1 \%$ BSA-BS $/ 0.05 \%$ Tween- 20 for 1 hour at $37^{\circ} \mathrm{C}$. This was followed by (incubation for 1 hour at $37^{\circ} \mathrm{C}$ with an optimal dilution) of one of the following biotinlabeled secondary rat anti-mouse antibodies: either anti-IgG1 (LO-MG1-15 from BioSource International, Camarillo, California, USA), anti-IgG2a (R19-15; PharMingen), or anti-IgE (2379-04S; BioSource International). Streptavidin-conjugated horseradish peroxidase was added for a 30-minute incubation at $37^{\circ} \mathrm{C}$, followed by addition of TMB substrate solution (DAKO Corp., Carpinteria, California, USA), and then termination of the reaction with $6 \mathrm{~N} \mathrm{HCl}$. Color change was read at $405 \mathrm{~nm}$ on a microplate ELISA reader (Bio-Tek Instruments Inc., Winooski, Vermont, USA). Antibody concentrations were calculated by comparison to standards: monoclonal antiOVA mouse IgG1 (clone OVA-14; Sigma Chemical Co.); monoclonal anti-OVA mouse IgE (kindly provided by E. Gelfand, National Jewish Center for Immunology and Respiratory Medicine, Denver, Colorado, USA); and pooled hyperimmune serum with high-titer anti-OVA IgG2a (concentration arbitrarily set at $200 \mathrm{U} / \mathrm{mL}$ ) generated by repeated intraperitoneal injection of $I L^{-4^{-/-}}$mice with OVA in alum.

Total serum IgE levels were measured in a similar ELISA with changes as follows. The coating was done with anti-mouse IgE (The Binding Site, Birmingham, United Kingdom) in borate saline at $4^{\circ} \mathrm{C}$ overnight; the standard was mouse IgE (IgE-3; PharMingen); and the biotin-labeled secondary antibody was rat anti-mouse IgE (LO-ME-3; BioSource International).

Statistical analyses. Statistical significance was determined using an unpaired Student's $t$ test.

\section{Results}

Th2 responses are induced by either e.c. or i.n. exposure to soluble OVA. Recent reports have demonstrated that prolonged e.c. exposure of mice to soluble protein preferentially induces $\mathrm{Th} 2$ activation $(31,32)$. We have also established Th2 responses in vivo by e.c. exposure of mice to soluble OVA under an occlusive skin patch. Mice exposed to e.c. OVA develop high levels of OVAspecific IgG1 and IgE but no detectable IgG2a in serum (Figure 1a). Furthermore, systemic Th2 activation was also evidenced in these mice by the type of inflammatory response generated in the airway upon challenge with i.n. OVA. As shown in Figure 1b, mice that had been epicutaneously sensitized and then challenged with i.n. OVA over a 6 -day period showed significant increases in both the total number of cells $(P=0.005)$ and the number of eosinophils $(P=0.0004)$ recovered by BAL, compared with unsensitized mice similarly exposed to i.n. OVA. In addition, PAS staining of lung sections demonstrated strong induction of PAS-positive mucus secretion in airways (see Figure 4a below). Other researchers have shown that overexpression of either IL-4 or IL-5 in the lung leads to increased mucus production $(33,34)$. Furthermore, studies from our laboratory using adoptive transfer of in vitro-generated Th1 or Th2 cells have established that recruitment of Th2 cells specifically to the airway is associated with eosinophilia and mucus hypersecretion, whereas Th1 cells are associated with neutrophils and little to no mucus production (35). Thus, both the high number of eosinophils and induction of mucus secretion in lungs after airway challenge of epicutaneously sensitized mice support the presence of Th2 effector cells. To confirm this, inflammatory cells were recovered from lung tissue by enzymatic digestion, and then restimulated in vitro with OVA. As shown in Figure 1c, the Th2 2 cytokines IL-4, IL-5, and IL-13 were all detected in culture supernatants, whereas IFN- $\gamma$, a Th1-associated cytokine, was not. Taken together, these data demonstrate highly skewed Th2 activation in our system after e.c. exposure to soluble OVA.

We also studied immune responses initially generated through the airway, for comparison with the epicutaneously induced Th2 responses described above. Primary exposure of mice to soluble i.n. OVA on 3 consecutive days also induced immune responses with 
characteristics of Th2 activation, as demonstrated by (a) high levels of OVA-specific IgG1 in serum (Figure 2a); (b) airway infiltrates with significantly higher numbers of eosinophils than were found in PBS-exposed controls after secondary airway challenge with OVA (Figure 2b; $P=0.04$ ); (c) production of Th2 cytokines (IL-4, IL-5, and IL-13) by lung inflammatory cells (Figure 2c); and

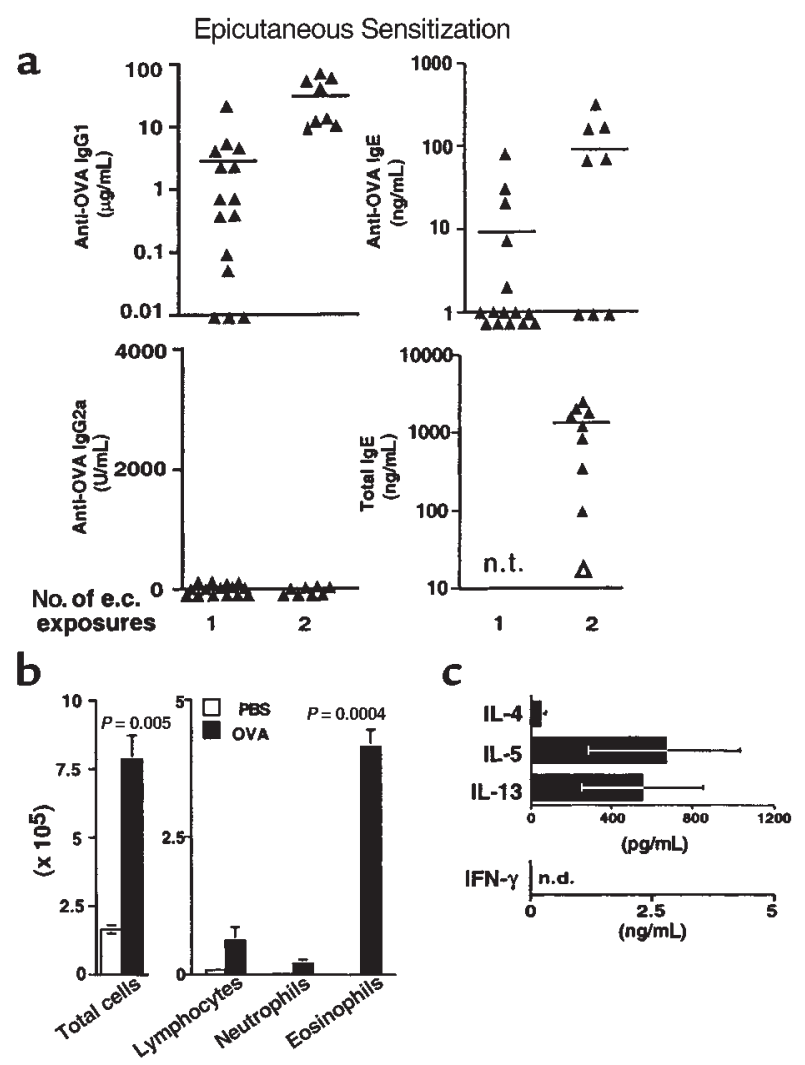

Figure 1

Th2 responses after e.c. OVA exposure. (a) Mice were exposed to e.c. OVA $(100 \mu \mathrm{g})$ under an occlusive skin patch either once (on day 0 ) or twice (on days 0 and 14). Serum was obtained for measurement of antibodies by ELISA on days 13 and 27. Data points represent values for individual mice with the mean indicated and are from 4 experiments. The open triangle at the bottom right represents the value for pooled preimmune serum. n.t., not tested. (b) Mice initially exposed epicutaneously to either OVA $(100 \mu \mathrm{g})$ in PBS or PBS alone on day 0 were challenged with i.n. OVA $(25 \mu \mathrm{g})$ on days $14,15,18$, and 19 . On day 21 , mice were sacrificed, BAL was performed, and total cell yield and differential counts in cytospin preparations from individual mice were determined. Both the total number of cells recovered by BAL and the number of each cell type are shown. Data are reported as mean \pm SEM of 3 (PBS) or 5 (OVA) mice per group. One representative experiment of 3 experiments with similar results is shown. Statistical significance between PBS-exposed and OVA-exposed groups was determined by unpaired Student's $t$ test. (c) After BAL on day 21, lungs from 5-10 mice initially exposed to e.c. OVA were harvested and pooled. Inflammatory cells were isolated by enzymatic digestion. Cells were restimulated in vitro by culture with OVA $(100 \mu \mathrm{g} / \mathrm{mL})$ for 48 hours. Cytokine levels in supernatants were measured by ELISA. Data are reported as mean \pm SEM from 2 experiments. n.d., none detected. Cytokine levels in cultures without OVA: IFN- $\gamma$, n.d.; IL-4, n.d.; IL$5,156 \mathrm{pg} / \mathrm{mL}$; IL-13, $59 \mathrm{pg} / \mathrm{mL}$. No cytokines were detected in cultures of lung cells from naive mice with or without OVA treatment. (d) strong PAS-positive staining of airways, demonstrating high amounts of mucus secretion (see Figure $4 \mathrm{e}$ below). In addition, although OVA-specific IgE was detectable in only a minority of the inhalation-sensitized mice, total IgE was elevated in all of them (Figure $2 a)$. It should be noted that Th2 skewing after inhalation priming was preferential - rather than absolute as seen in epicutaneously sensitized mice - in that IFN- $\gamma$ was also detected in cultures of lung inflammatory cells (Figure 2c), and some mice demonstrated OVA-specific IgG2a responses, an isotype associated with IFN- $\gamma$ production by Th 1 cells (Figure 2a). However, neither the preferential Th2 induction by i.n. sensitization nor the Th2 responses generated after e.c. OVA exposure could be attributed to an antigen dose effect. Dose-response studies performed using a wide range of OVA $(0.1-1,000$ $\mu \mathrm{g})$ for both i.n. and e.c. sensitization showed no change in the type of response induced with different OVA doses. Serum antibody levels and total BAL fluid numbers did rise with increasing OVA dose, but the antibody isotype pattern and high percentage of eosinophils in BAL fluid remained the same (not shown). Optimal responses in this study were reached with the $100-\mu \mathrm{g}$ sensitizing dose. In summary, the ability to generate strong Th2 activation in the absence of an adjuvant such as alum, at both these anatomic sites that would normally encounter environmental allergens, supports the particular relevance of using these models for investigation of the critical immunoregulatory factors involved in atopic responses.

IL-4-deficient mice show impaired Th2 responses after primary i.n. OVA exposure. Our goal was to investigate the importance of IL-4 in generation of Th2 responses at sites where environmental allergens are normally encountered, namely the skin and airway. $I L^{-4^{-/-}}$mice were initially exposed to either e.c. or i.n. OVA as above, and then challenged with OVA via the airway. As shown in Figure 3, although airway inflammatory responses were still induced in $I L^{-4^{-/}}$mice initially exposed to i.n. OVA, these responses had characteristics of predominant Th1 activation, with little evidence of the Th2 responses seen in wild-type (WT) mice. Specifically, the number of eosinophils in BAL fluid was significantly reduced in $I L^{-4^{-/}}$mice compared with WT mice $(P=$ 0.0007), whereas the number of neutrophils was modestly increased (Figure 3a). Furthermore, mucus secretion, another indicator of Th2 activation, was diminished in lungs of inhalation-primed $I L-4^{-/-}$mice, as indicated by decreased PAS staining of airways compared with WT controls (Figure 4, g and e, respectively). This was despite equivalent amounts of inflammation being present in the lungs of $I L^{-4^{-/-}}$mice and WT mice, as judged by scoring of histologic sections (not shown; see Methods for description of histologic inflammation index). Histologic examination also revealed a marked reduction in eosinophils in lungs of inhalation-sensitized $I L-4^{-/-}$mice compared with WT mice, which further supports a shift toward Th1 activation. Likewise, the antibody isotype profile in serum of $I L-4^{-/-}$mice was 
Figure 2

Th2 responses after i.n. OVA exposure. Mice received an initial course of i.n. OVA ( $100 \mu \mathrm{g}$ on day $0-2)$, and then a second challenge course (25 $\mu \mathrm{g}$ on days $14,15,18$, and 19). (a) Serum was obtained for measurement of antibodies by ELISA on days 13 and 21. Data points represent values for individual mice with the mean indicated, and are from 2 experiments. The open triangle at bottom right represents the value for pooled preimmune serum. (b) Mice initially exposed to either i.n. OVA $(100 \mu \mathrm{g})$ in PBS or PBS alone on days $0-2$ were challenged with i.n. OVA $(25 \mu \mathrm{g})$ on days $14,15,18$, and 19 . On day 21 , mice were sacrificed, BAL was performed, and total cell yield and differential counts in cytospin preparations from individual mice were determined. Both the total number of cells recovered by BAL and the number of each cell type are shown. Data are reported as mean \pm SEM of 3 (PBS) or 5 (OVA) mice per group. One representative experiment of 3 experiments with similar results is shown. Statistical significance between PBS-exposed and OVA-exposed groups was determined by unpaired Student's $t$ test. (c) After BAL on day 21, lungs from 5-10 mice initially exposed to i.n. OVA were harvested and pooled. Inflammatory cells were isolated by enzymatic digestion. Cells were restimulated in vitro by culture with OVA $(100 \mu \mathrm{g} / \mathrm{mL})$ for 48 hours, and cytokine levels in supernatants were measured by ELISA. Data are reported as mean \pm SEM from 2 experiments. Cytokine levels in cultures without OVA: IFN- $\gamma$, n.d.; IL-4, n.d.; IL-5, $135 \mathrm{pg} / \mathrm{mL}$; IL-13, n.d. No cytokines were detected in cultures of lung cells from naive mice with or without OVA treatment. a

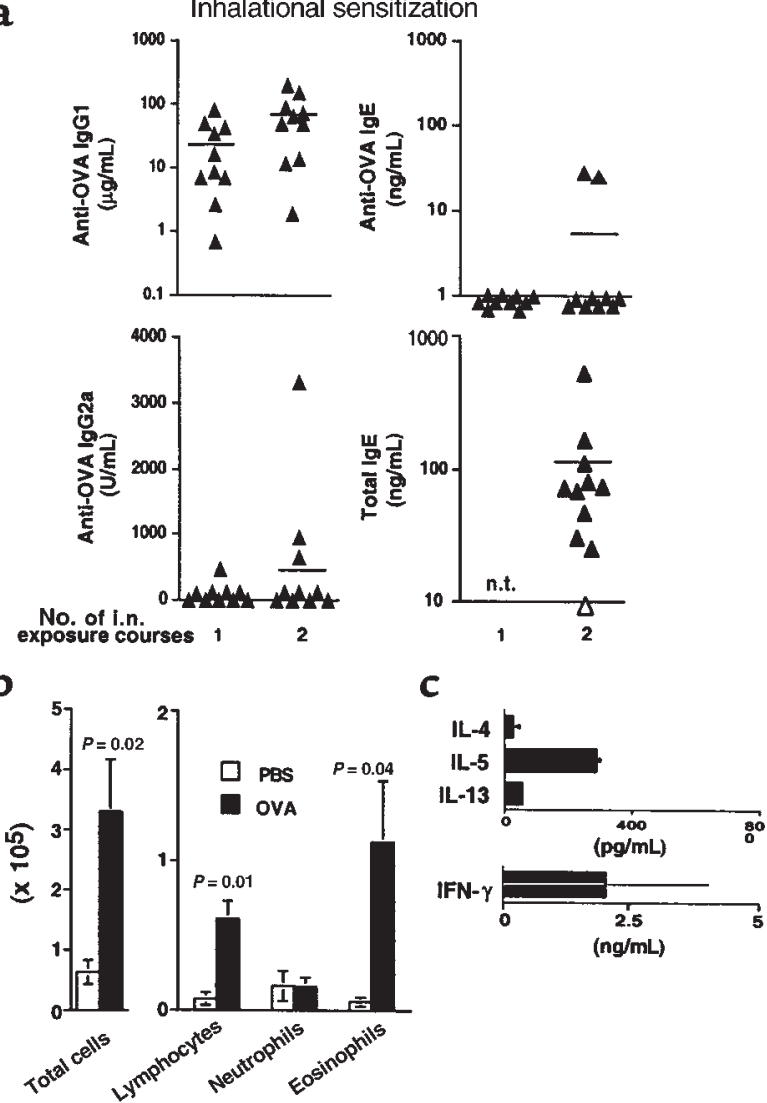

consistent with a Th1 response, with little to no antiOVA IgG1 and no IgE, but high levels of OVA-specific IgG2a (Figure 3b). Therefore, in the absence of IL-4, i.n. OVA exposure led to a predominantly Th1 response.

Th2 responses are still induced by primary e.c. OVA exposure in $I L^{-4^{-/-}}$mice. In contrast to the loss of Th2 activation in $I L-$ $4^{-/-}$mice after primary i.n. sensitization, e.c. sensitization of $I L-4^{-/-}$mice resulted in strong Th2 activation. Airway inflammatory responses induced by OVA challenge of epicutaneously sensitized $I L^{-4^{-/-}}$mice were characterized by high numbers of eosinophils, equivalent to those seen in WT mice (Figure 5a). Mucus hypersecretion, as indicated by strongly positive PAS staining of airways, was also present (Figure 4c). Of note, persistence of an eosinophilic response in lungs of epicutaneously sensitized, as opposed to inhalation-sensitized, $\mathrm{IL}^{-4^{-/}}$mice was not a reflection of differences in the baseline eosinophil numbers present in lung or BAL fluid before airway challenge. Mice that had been either epicutaneously or inhalation sensitized, but not challenged with OVA, had similar total numbers of cells in BAL fluid on day 14: $(1.2 \pm 0.3) \times 10^{5}$ and $(1.0 \pm$ $0.1) \times 10^{5}$, respectively. Furthermore, there was no increase in eosinophils in BAL fluid of mice sensitized by e.c. or i.n. OVA on day 14 compared with PBS-exposed mice (fewer than $0.01 \times 10^{5}$ eosinophils in all groups), nor was there any detectable increase in eosinophils in lung tissue by histologic analysis (not shown).

Analysis of OVA-specific antibodies in serum of epicutaneously sensitized $I L^{-4^{-/-}}$mice did show a somewhat altered isotype profile, with reduced levels of OVA-spe- cific IgG1 and IgE, and higher levels of anti-OVA IgG2a (Figure 5b). The reduction in IgE observed in $\mathrm{IL}_{-4}^{-/-}$mice is not surprising, given that IL-4 is the major stimulus for isotype class switching to IgE. Thus, even if Th 2 cells secreting IL-5 and IL-13 are present, they will not be able to stimulate high IgE levels without IL-4. Although the presence of OVA-specific IgG2a was variable in epicutaneously sensitized $I L_{-4^{-/}}$mice, we have never detected anti-OVA IgG2a in epicutaneously sensitized WT mice $(n>30)$. This raises the possibility that in the absence of IL-4, some Th1 activation may be occurring after e.c. OVA exposure. However, the cellular composition of BAL fluid from these mice does not support this, because equivalent numbers of neutrophils and eosinophils were present in WT and $I L^{-4^{-/}}$mice (Figure 5a). Thus, if Th1 activation had occurred, Th1 effector cells either were not recruited to the lung after i.n. OVA challenge, or were not at a level that would affect the Th2 response. Alternatively, higher IgG2a levels present in $I L$ $4^{-/-}$mice may simply reflect the specific lack of IL-4, which by decreasing class switching to IgG1 and IgE, may shift the balance to IgG2a.

To clarify the issue of whether Th1 effector cells, along with Th2 cells, were present in the lungs of epicutaneously sensitized $I L-4^{-/-}$mice after airway challenge, further analysis of lung tissue infiltrates was undertaken. The overall amount of inflammation present in lung sections was first assessed using a histologic index based on the number of airways and vascular profiles surrounded by inflammatory cells, and the density of these 
a

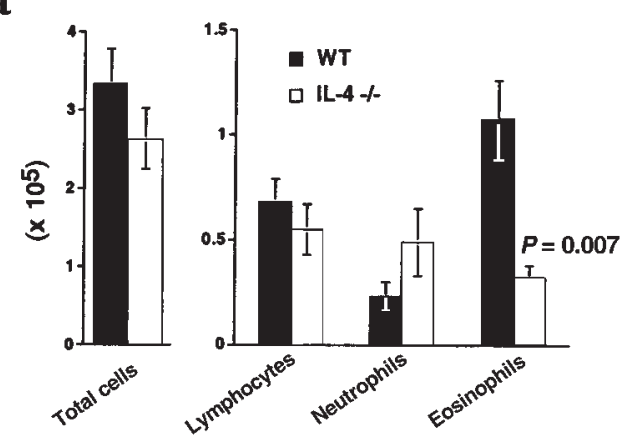

b
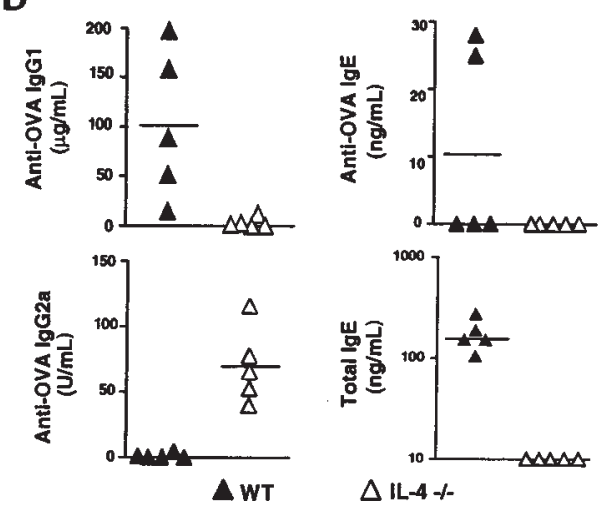

Figure 3

Airway inflammatory responses and serum antibody production in i.n. OVA-sensitized $I L-4^{-/-}$mice. C57BL/ 6 (WT) or $I L-4^{-/-}$mice were initially exposed to i.n. OVA $(100 \mu \mathrm{g})$ on days $0-2$, and then challenged with i.n. OVA $(25 \mu \mathrm{g})$ on days $14,15,18$, and 19. (a) On day 21 , mice were sacrificed, BAL was performed, and total cell yield and differential counts in cytospin preparations from individual mice were determined. Data were pooled from 2 experiments and are reported as mean \pm SEM of $8(\mathrm{WT})$ or $9\left(\mathrm{IL}-4^{-/-}\right)$mice per group. Both the total number of cells recovered by BAL and the number of each cell type are shown. Statistical significance between WT and $I L-4^{-/-}$groups was determined by unpaired Student's $t$ test. (b) Serum was obtained for measurement of antibodies by ELISA on day 21. Data points represent values for individual mice with the mean indicated, and are from 1 representative experiment of 2 with similar results.

infiltrates. As shown in Figure 6a, no detectable difference was seen between WT and $I L^{-4^{-/}}$mice in the amount of inflammation present in lungs after e.c. sensitization and airway challenge with OVA. Lung inflammatory cells were then isolated to determine relative numbers of T-cell subsets and cytokine secretion profiles. After initial e.c. OVA sensitization and subsequent i.n. OVA challenge of either WT or $I L^{-4^{-/-}}$mice, lungs from several mice were pooled and inflammatory cells were isolated by enzymatic digestion. As shown in Figure 6b, equivalent numbers of cells were isolated from lungs of WT and $I L^{-4^{-/-}}$mice, with similar numbers of CD 4 and CD8 T cells present in the infiltrates of both groups. These cells were then restimulated in vitro by culture with OVA to determine their cytokine production profiles. Cells isolated from lungs of either WT mice or $I L^{-4^{-/-}}$mice that had been epicutaneously sensitized with OVA before airway challenge secreted high levels of the Th2 cytokines IL-5 and IL-13 that were sta- tistically indistinguishable, although the levels of both cytokines were somewhat lower in $I L-4^{-/-}$cultures than in WT cultures (Figure 6c). These data confirm that Th2 activation did occur in epicutaneously sensitized $\mathrm{IL}_{-} 4^{-/-}$ mice. In contrast, no IFN- $\gamma$ was detected in supernatants of $\mathrm{IL}^{-4^{-/}}$lung inflammatory cell cultures. Thus, although the presence of OVA-specific IgG2a suggests that Th1 activation may have occurred in these mice, Th1 cells were either not recruited to the lung as effectively as were Th2 cells, or were not present in high enough numbers to be detectable.

STAT6 signaling is required for generation of Th2 responses after e.c. OVA exposure. The ability of IL-4 to induce differentiation of naive CD4 T cells into Th2 effector cells is critically linked to its ability to activate the STAT6 intracellular signaling pathway (36-38). In other words, STAT6 activation itself, whether caused by IL-4 or another cytokine, is more precisely the true inducer of Th2 generation. We predicted, therefore, that the IL4 -independent Th2 responses induced by e.c. OVA exposure in our model would retain their dependence on STAT6 signaling. To test this hypothesis, mice deficient in STAT6 (Stat6 ${ }^{--}$) were exposed to e.c. OVA and subsequently challenged with i.n. OVA as described previously. Epicutaneously sensitized Stat6 ${ }^{-/}$mice did mount airway inflammatory responses after i.n. OVA challenge, with significant increases in total BAL fluid cell numbers compared with PBS-exposed controls $\left[(6.4 \pm 1.1) \times 10^{5}\right.$ vs. $(1.2 \pm 0.2) \times 10^{5}$, respectively; $P=$ 0.002 ; not shown]. However, as shown in Figure 7, these responses had characteristics of Th1, rather than Th2, activation, with significantly reduced numbers of eosinophils $(P<0.0001)$ and increased numbers of neutrophils $(P=0.01)$ in BAL fluid compared with WT controls. Loss of Th2 activation was further supported by absence of PAS-positive staining of airways of inflamed lungs from $\mathrm{Stat}^{-/-}$mice (not shown). Not surprisingly, the antibody isotype profile in serum of epicutaneously sensitized $\mathrm{Stat}^{-/-}$mice also indicated Th1 activation by the marked decrease in OVA-specific IgG1 (12 \pm 4.6 $\mu \mathrm{g} / \mathrm{mL} ; n=7)$ compared with WT controls $(137.9 \pm 44.6$ $\mu \mathrm{g} / \mathrm{mL} ; n=5)$. In contrast, IgG2a levels were increased (34.8 $\pm 9 \mathrm{U} / \mathrm{mL}$ vs. undetectable, respectively). Thus, in contrast to a lack of requirement for IL-4, epicutaneously induced Th2 responses were dependent on STAT6 signaling. These data suggest that Th2 responses generated by e.c. sensitization of $I L^{-4^{-/}}$mice in this study resulted from activation of STAT6 by a factor other than IL-4.

Th2 responses are no longer generated after primary e.c. OVA exposure of $I L^{-4^{-/}}$mice depleted of $I L-13$. As stated above, the STAT6 dependence of Th2 responses generated by e.c. OVA exposure in our system suggested that epicutaneously induced Th2 responses present in IL-4/- mice must involve activation of STAT 6 by some factor other than IL-4. We hypothesized that this factor was likely to be IL-13, a Th2-associated cytokine that shares many biologic functions with IL-4, including the ability to activate STAT6 $(39,40)$. To investi- 


\section{Figure 4}

PAS staining of lung sections $(\times 200)$ from epicutaneously or inhalation-sensitized mice after airway challenge with OVA. Mice were initially exposed to either e.c. or i.n. OVA or PBS. Beginning on day 14, all mice were challenged with i.n. OVA over a 6-day period, and lungs were harvested for histologic analysis on day 21.

gate the role of IL-13 in our system, $I L$ $4^{-/-}$mice were depleted of IL-13 in vivo by repeated administration of an IL-13 antagonist (IL-13R $\alpha-\mathrm{Fc}) . I L-4^{-/-}$mice, depleted or not of IL-13, were epicutaneously sensitized and then challenged with OVA through the airway. As shown in Figure 8, mice treated with IL-13R $\alpha$ Fc had significantly fewer eosinophils in BAL fluid than did mice receiving the control Fc $(P=0.009)$, whereas the numbers of neutrophils were slightly increased. These results are consistent with a loss of the Th2 response, and a switch toward Th1 activation, as had been seen in $\mathrm{Stat}^{-/-}$mice. PAS-positive staining of airways was also absent in mice treated with the IL-13R $\alpha$-Fc antagonist (not shown). These data show that IL-13 is critical for the maintenance of Th2 activation in $I L^{-4^{-/}}$mice after primary e.c. OVA exposure.

\section{Discussion}

A critical requirement for developing new treatment strategies for atopic disease is better understanding of which immunoregulatory factors are responsible for the hallmark predisposition toward Th2 activation. The goal of this study was to assess the importance of IL-4, a known Th2-promoting cytokine, in generation of an allergic-type Th2 response through use of mouse models that closely mimic natural allergen exposure. Using $I L_{-4^{-/}}$mice, we investigated the role of IL- 4 in Th2 responses induced by initial exposure of either the skin or airway to soluble protein, and identified a marked difference in the IL-4 requirement at these two sites. Th 2 responses generated by initial airway exposure to OVA were highly dependent on IL-4, whereas epicutaneously induced Th2 responses were not. Instead, IL-13 was sufficient to generate Th2 responses after e.c. OVA exposure. The data provide particularly relevant insight into the potential for distinct Th2 activation requirements at two sites of environmental allergen encounter - the skin and lung - and suggest that the cutaneous microenvironment may more readily promote $\mathrm{Th} 2$ sensitization than was previously appreciated.

In our models, initial exposure of WT mice to soluble OVA either by inhalation or skin contact, in the

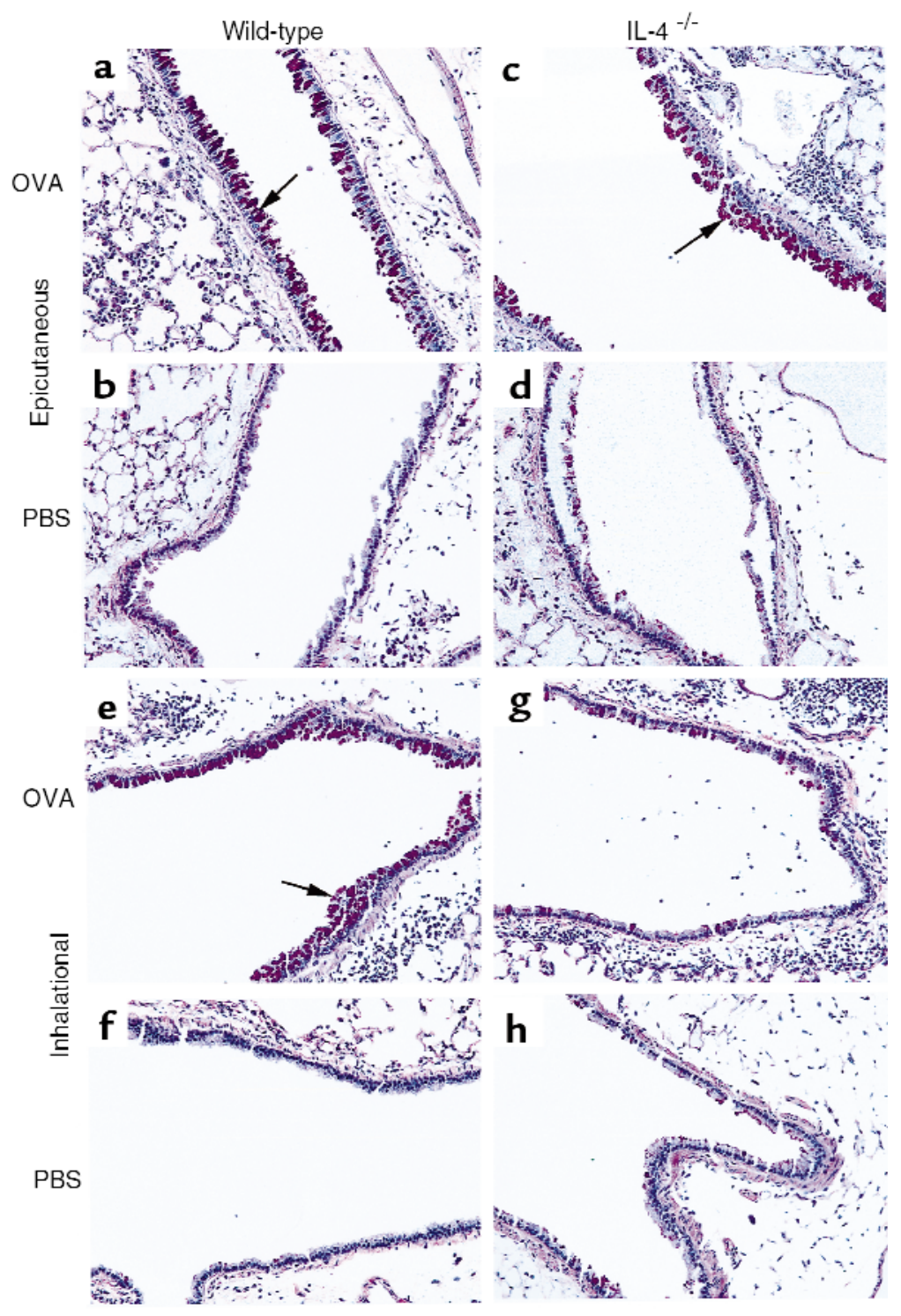

absence of adjuvant, induced strong Th2 responses. This was demonstrated by a characteristic Th2-type antibody isotype profile in serum, with high levels of $\operatorname{IgE}$ and $\operatorname{IgG} 1$ (both induced by IL-4), and little IgG2a, the murine isotype associated with IFN- $\gamma$ production by Th1 cells $(41,42)$. In addition, subsequent airway challenge of either epicutaneously or inhalation-sensitized mice led to lung responses with characteristics of Th2 recruitment (35), including inflammatory infiltrates with high numbers of eosinophils, mucus hypersecretion, and production of Th2 cytokines (IL-4, IL-5, and IL-13) by lung inflammatory cells. The generation of robust Th2 responses after exposure of the skin to soluble OVA in our study is in agreement with other studies $(31,32)$. We also report generation of Th2 responses after primary exposure of mice to OVA by inhalation, a finding that has not been widely reported in the literature. In fact, several studies examining the effects of primary aerosol exposure to protein have reported suppression or tolerance induction via this 
Figure 5

Airway inflammatory responses and serum antibody production in e.c. OVA-sensitized IL-4-/- mice. C57BL/ $6 \mathrm{WT}$ or $/ \mathrm{L}-4^{-/-}$mice were initially exposed to e.c. OVA $(100 \mu \mathrm{g})$ on day 0 , and then challenged with i.n. OVA $(25 \mu \mathrm{g})$ on days $14,15,18$, and 19. (a) On day 21 , mice were sacrificed, BAL was performed, and total cell yield and differential counts in cytospin preparations from individual mice were determined. Data were pooled from 2 experiments and are reported as mean \pm SEM of 6 (WT) or $7\left(\mathrm{IL}-4^{-/-}\right)$mice per group. Both the total number of cells recovered by BAL and the number of each cell type are shown. No statistically significant differences were found between WT and IL-4-/groups as determined by unpaired Student's $t$ test. (b) Serum was obtained for measurement of antibodies by ELISA on day 21. Data points represent values for individual mice with the indicated mean, and are from 1 representative experiment of 2 with similar results.

route $(43,44)$. One group, however, has reported effective sensitization of mice after exposure to aerosolized protein (45). Subtle differences in the dose of antigen used or kinetics of delivery may explain the discrepancies in the literature. With regard to this study, the dose and time course of i.n. OVA exposure used consistently resulted in Th2 generation.

Establishment of vigorous Th2 responses by primary exposure of mice to soluble OVA either through the skin or airway provides two particularly relevant models of allergen-induced Th2 activation, and studies aimed at addressing the role of IL-4 in these responses revealed interesting differences between these two sites of sensitization. After initial i.n. OVA exposure, $I L^{-4^{-/}-}$ mice showed diminished Th2 responses compared with WT controls, with subsequent airway challenge leading to infiltrates containing markedly decreased numbers of eosinophils and minimal mucus secretion in airways. Instead, the inflammatory responses seen in these mice had characteristics of Th1 effector cell recruitment, with higher numbers of neutrophils in BAL fluid than were found in WT controls. A shift toward Th1 activation a

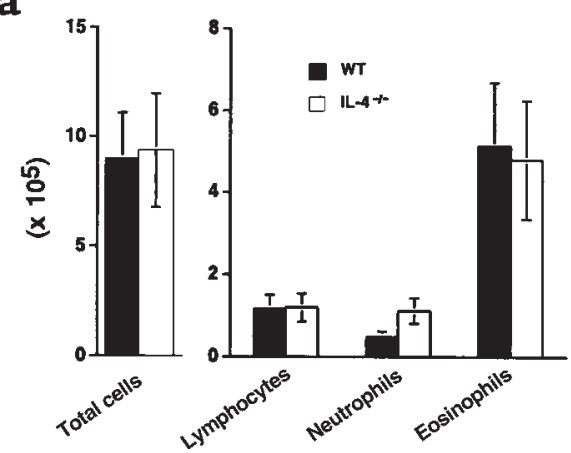

b
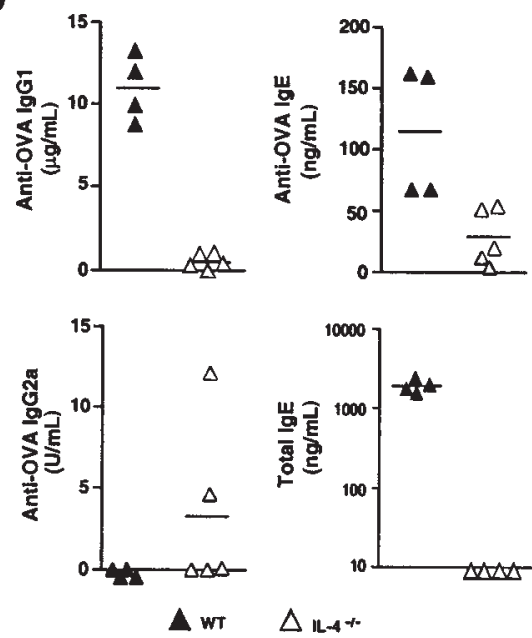

was also reflected in the antibody isotype profile, with $I L-4^{-/}$mice showing much lower OVA-specific IgG1 responses and higher IgG2a responses. These data are consistent with published results of many other researchers who have examined responses to protein antigens in $I L^{-4^{-/-}}$mice, and reported either absent or markedly impaired Th2 responses $(16,19,21,46,47)$.
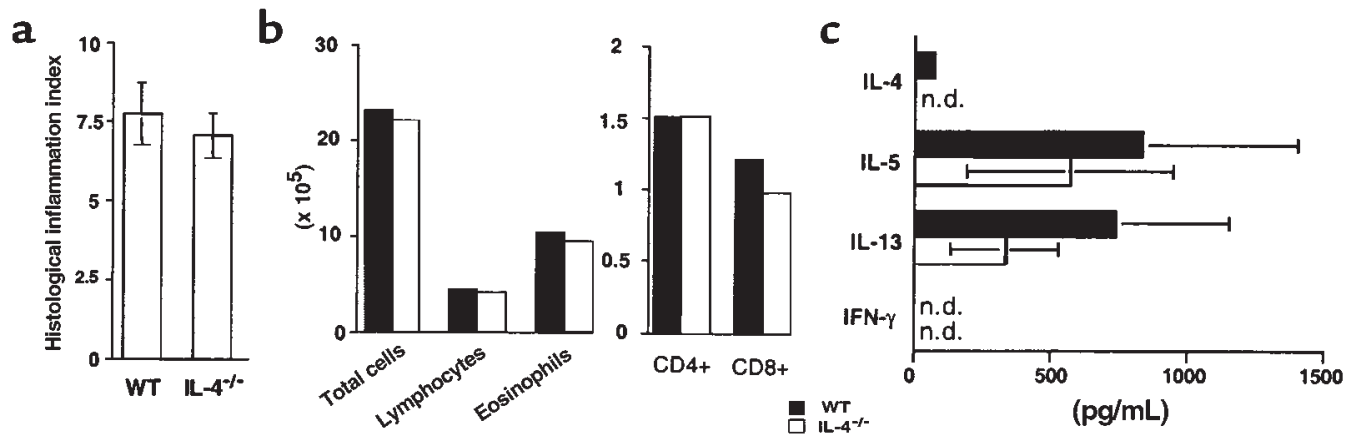

\section{Figure 6}

Comparison of lung inflammatory responses after airway challenge of epicutaneously sensitized WT or IL-4 ${ }^{-/-}$mice. C57BL/6 WT or IL-4 ${ }^{-/-}$ mice were initially exposed to e.c. OVA $(100 \mu \mathrm{g})$ on day 0 , challenged with i.n. OVA ( $25 \mu \mathrm{g})$ on days $14,15,18$, and 19 , and sacrificed on day 21. (a) In some experiments, lungs were harvested, processed for routine histologic analysis (H\&E staining), and scored for inflammation (see Methods). Data are from 3 experiments and are reported as the mean \pm SEM of 11 (WT) or 13 (IL-4 $4^{-/}$) mice per group. (b and $\mathbf{c}$ ) In other experiments, lungs were harvested from 5-10 mice per group and pooled. Inflammatory cells were isolated by enzymatic digestion. Cells were then either analyzed by flow cytometry or restimulated in vitro by culture with OVA ( $100 \mu \mathrm{g} / \mathrm{mL})$ for 48 hours, and cytokine levels were measured in supernatants by ELISA. Data are reported as the number of cells isolated per mouse from a representative experiment (b), or as the mean \pm SEM cytokine concentration in culture supernatants from 2 experiments (c). 


\section{Figure 7}

Cellular composition of BAL fluid after airway challenge of epicutaneously sensitized Stat6 ${ }^{-/-}$mice. C57BL/ 6 WT or Stat $6^{-/-}$mice were initially exposed to e.c. OVA $(100 \mu \mathrm{g})$ on day 0 , and then challenged with i.n. OVA ( $25 \mu \mathrm{g})$ on days $14,15,18$, and 19 . On day 21 , mice were sacrificed, BAL was performed, and total cell yield and differential counts in cytospin preparations from individual mice were determined. Data were pooled from 2 experiments and are reported as mean \pm SEM of both the total number of cells recovered by BAL and the number of each cell type. Statistical significance between WT $(n=9)$ and Stat6 ${ }^{-1-}(n=7)$ groups was determined by unpaired Student's $t$ test.
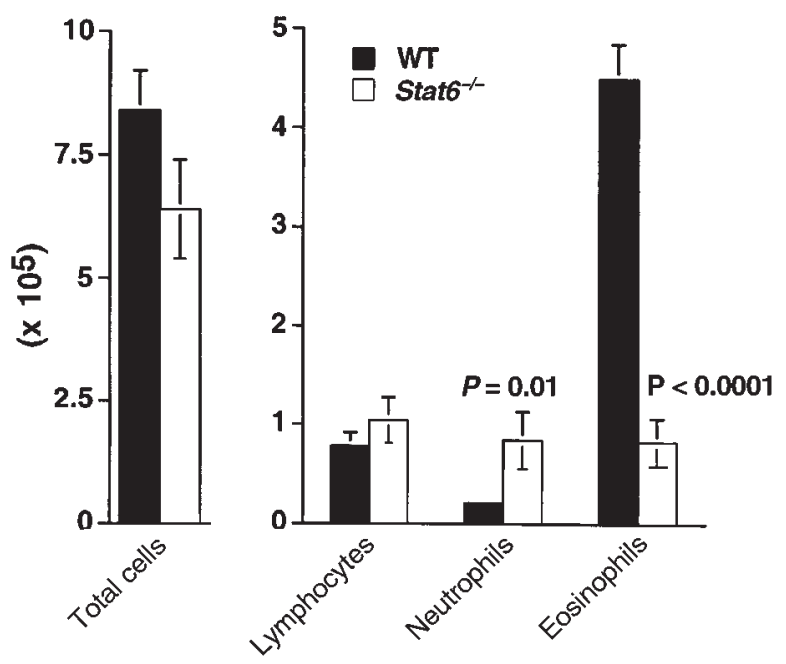

recently reported impaired eosinophil recruitment into inflamed skin sites in $\mathrm{IL}_{-4^{-/-}}$mice using a model of e.c. protein sensitization similar to that described here (47). In their study, the local inflammatory response induced in skin after repeated e.c. exposures to soluble OVA was evaluated. Whereas WT mice had increased numbers of eosinophils at these sites, the inflammatory infiltrates in $I L^{-4^{-/}}$mice had significantly fewer eosinophils. In our study, however, we saw no deficit in recruitment of eosinophils into the airway after inhalation challenge of epicutaneously sensitized $I L-4^{-/-}$mice. This difference probably reflects distinct requirements for leukocyte emigration into tissue at these two sites of antigen challenge, i.e., the skin vs. the lung. Several factors contribute to leukocyte trafficking across microvascular endothelium, including various proinflammatory cytokines and specific interactions between adhesion molecules expressed by endothelial cells and their ligands on the surface of inflammatory cells. Thus, whereas cytokines secreted by $I L^{-4^{-/-}}$Th2 effector cells, including IL-5 and IL-13, may be sufficient for initiation of an inflammatory response and recruitment of eosinophils into the lung, control of leukocyte migration into skin may be more specifically dependent on IL-4. With regard to eosinophils in
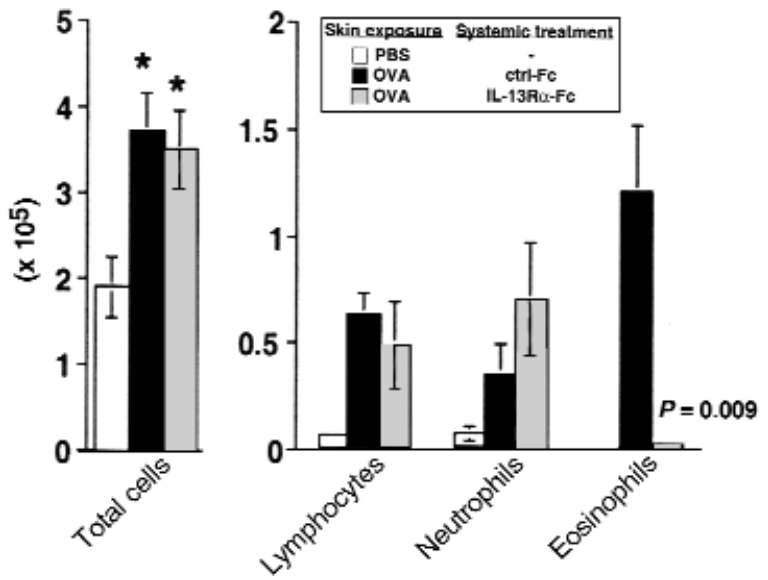

\section{Figure 8}

Cellular composition of BAL fluid after airway challenge of epicutaneously sensitized $/ \mathrm{L}-4^{-/-}$mice depleted of IL-13 in vivo. $/ \mathrm{L}-4^{-/-}$ mice were initially exposed epicutaneously to either OVA $(100 \mu \mathrm{g})$ in PBS or PBS alone on day 0 , and then challenged with i.n. OVA ( 25 $\mu \mathrm{g}$ ) on days $14,15,18$, and 19 . OVA-exposed mice also received intraperitoneal injections of either an IL-13 antagonist (IL-13R $\alpha-F \mathrm{C}$ ) or the Fc control (ctrl-Fc) every other day, from day -1 to day 19. On day 21 , mice were sacrificed, BAL was performed, and total cell yield and differential counts in cytospin preparations from individual mice were determined. Data are reported as mean \pm SEM (3 mice per group) of both the total number of cells recovered by BAL and the number of each cell type. Statistical significance between groups was determined by unpaired Student's $t$ test. ${ }^{*} P<0.05$ for PBS-exposed vs. OVA-exposed groups; $P=0.009$ for eosinophils IL$13 R \alpha-F c$ vs. ctrl-Fc-treated groups. 
particular, interaction of the $\alpha 4$-integrin VLA- 4 on the eosinophil surface with its ligand VCAM-1 on endothelial cells is very important in allowing egress of eosinophils into inflamed tissue (48). Furthermore, IL4 can upregulate VCAM-1 expression on endothelial cells (49). Thus, a lack of IL-4 could result in decreased VCAM-1 and a subsequent defect in eosinophil migration. However, Spergel et al. reported no detectable difference in VCAM-1 staining of epicutaneously exposed skin sites between WT mice and $I L-4^{-/-}$mice (47). Still, the possibility remains that another IL-4-dependent adhesive interaction exists that is needed for eosinophils to migrate across cutaneous, but not pulmonary, microvasculature.

The role of IL-4 in Th2 skewing is tightly linked to its ability to activate the STAT6 intracellular signaling pathway $(36,37)$. Binding of IL-4 to the IL-4 receptor $\alpha$ chain (IL-4R $\alpha$ ) results in phosphorylation of STAT6; studies by several groups have established that the ability to generate Th2 responses is markedly hampered in Stat $6^{-1-}$ mice $(38,50-52)$. Our finding of strikingly impaired Th2 activation after e.c. OVA exposure of Stat $6^{-/}$mice is in agreement with these studies, and confirms that the Th2 responses in our system also work via the STAT6 signaling pathway. It follows that a factor other than IL-4 must be capable of inducing STAT6 activation in our system, because effective Th2 activation did occur after e.c. OVA exposure of $I L^{-4^{-/}-}$ mice. IL-13 was a likely candidate because it too can induce activation of STAT 6 by binding to the highaffinity IL-13 receptor complex, one component of which is the IL-4R $\alpha$ chain $(39,40,53,54)$. Moreover, it has recently been found that IL-13 is the factor responsible for IL-4-independent Th2 generation in $\mathrm{IL}_{-4} 4^{-/}$ mice infected with Nippostrongylus $(28,29)$. A major role for IL-13 in allergic airway inflammatory responses in mice has also been demonstrated $(55,56)$. To confirm that IL-13 was the STAT6-inducing factor mediating IL-4-independent Th2 activation in our system after e.c. sensitization, we blocked IL-13 activity in vivo by administration of an IL-13 antagonist. As expected, depletion of IL-13 in epicutaneously sensitized $I L^{-4^{-/}}$ mice resulted in a loss of Th2 activation, indicating that IL-13 could effectively stimulate generation of a Th2 response after e.c. protein exposure, even in the absence of IL-4. It should be noted that unlike human $\mathrm{T}$ cells, murine $\mathrm{T}$ cells have not been shown to respond to IL-13 (39). Therefore, the exact mechanism by which IL-13 mediates Th2 activation in our system and in other models (27-29) remains unclear. It may be that expression of IL-13 receptors by murine T cells occurs only under specific conditions of T-cell activation in vivo that have yet to be duplicated ex vivo or in vitro. Alternatively, Th2 differentiation may occur as an indirect effect after IL-13-induced STAT6 activation of another cell population that responds to IL-13, such as macrophages or dendritic cells $(38,57,58)$.

In summary, this study describes an interesting dichotomy in the IL-4 dependence of Th2 responses generated by exposure of two different anatomic sites to soluble protein antigen. Whereas induction of Th2 inflammatory responses by primary exposure to i.n. antigen was significantly impaired in $\mathrm{IL}_{-4^{-/-}}$mice, Th2 responses equivalent to those in WT mice were still generated in $I L^{-4^{-1}}$ mice after e.c. antigen exposure. The lack of Th2 activation in Stat $6^{-1-}$ mice after e.c. sensitization indicated that a factor other than IL-4, probably IL-13, was capable of inducing STAT6 activation in epicutaneously sensitized $I L-4^{-/-}$mice. Indeed, in vivo depletion of IL-13 in epicutaneously sensitized $I L^{-4^{-/-}}$ mice did result in a loss of the Th2 response. This study provides particularly relevant information with regard to the immunoregulatory factors controlling Th2 activation at sites of natural allergen exposure, namely the skin and lung. In addition, the data point to the skin as a powerful site for Th2 activation, even in the absence of IL-4, and raise new questions about its relative contribution in early sensitization of atopic individuals.

\section{Acknowledgments}

The authors would like to thank S. Constant and K. Steger for critical review of the manuscript, and Debra Donaldson at Genetics Institute Inc. for providing the soluble IL-13R $\alpha$-Fc fusion protein. This work was supported by National Institutes of Health grant R01-HL54450 (K. Bottomly) and a pilot/feasability project award from the Yale Skin Diseases Research Core Center (P30-AR4192) (C. Herrick). C. Herrick was supported by National Institutes of Health Training Grant T32-AR-07016 and by a Dermatology Foundation Dermatologist Investigator Fellowship.

1. van Reijsen, F.C., et al. 1992. Skin-derived aeroallergen-specific T-cell clones of Th2 phenotype in patients with atopic dermatitis. J. Allergy Clin. Immunol. 90:184-192.

2. Hamid, Q., Boguniewicz, M., and Leung, D.Y. 1994. Differential in situ cytokine gene expression in acute vs. chronic atopic dermatitis. J. Clin. Invest. 94:870-876.

3. Hamid, Q., et al. 1996. In vivo expression of IL-12 and IL-13 in atopic dermatitis. J. Allergy Clin. Immunol. 98:225-231.

4. Robinson, D., et al. 1993. Activation of CD4+ T cells, increased TH2-type cytokine mRNA expression, and eosinophil recruitment in bronchoalveolar lavage after allergen inhalation challenge in patients with atopic asthma. J. Allergy Clin. Immunol. 92:313-324.

5. Robinson, D.S., et al. 1992. Predominant TH2-like bronchoalveolar T-lymphocyte population in atopic asthma. N. Engl.J. Med. 326:298-304.

6. Huang, S.K., et al. 1995. IL-13 expression at the sites of allergen challenge in patients with asthma. J. Immunol. 155:2688-2694.

7. Bonnefoy, J.Y., Gauchat, J.F., Lecoanet-Henchoz, S., Graber, P., and Aubry, J.P. 1996. Regulation of human IgE synthesis. Ann. NY Acad. Sci. 796:59-71.

8. Finkelman, F.D., et al. 1988. IL-4 is required to generate and sustain in vivo IgE responses. J. Immunol. 141:2335-2341.

9. Resnick, M.B., and Weller, P.F. 1993. Mechanisms of eosinophil recruitment. Am. J. Respir. Cell Mol. Biol. 8:349-355.

10. Constant, S.L., and Bottomly, K. 1997. Induction of Th1 and Th2 CD4+ T cell responses: the alternative approaches. Annu. Rev. Immunol. 15:297-322.

11. Constant, S., Pfeiffer, C., Woodard, A., Pasqualini, T., and Bottomly, K. 1995. Extent of $\mathrm{T}$ cell receptor ligation can determine the functional differentiation of naive CD4+ T cells. J. Exp. Med. 182:1591-1596.

12. Seder, R.A., and Paul, W.E. 1994. Acquisition of lymphokine-producing phenotype by CD4+ T cells. Annu. Rev. Immunol. 12:635-673.

13. Abbas, A.K., Murphy, K.M., and Sher, A. 1996. Functional diversity of helper T lymphocytes. Nature. 383:787-793.

14. Swain, S.L., Weinberg, A.D., English, M., and Huston, G. 1990. IL-4 directs the development of Th2-like helper effectors. J. Immunol. 145:3796-3806.

15. Seder, R.A., Paul, W.E., Davis, M.M., and Fazekas de St. Groth, B. 1992. The presence of interleukin 4 during in vitro priming determines the lymphokine-producing potential of CD4+ $\mathrm{T}$ cells from $\mathrm{T}$ cell receptor transgenic 
mice. J. Exp. Med. 176:1091-1098.

16. Kopf, M., et al. 1993. Disruption of the murine IL-4 gene blocks Th2 cytokine responses. Nature. 362:245-248.

17. Kuhn, R., Rajewsky, K., and Muller, W. 1991. Generation and analysis of interleukin-4 deficient mice. Science. 254:707-710.

18. Noben-Trauth, N., Kropf, P., and Muller, I. 1996. Susceptibility to Leishmania major infection in interleukin-4-deficient mice. Science. 271:987-990.

19. Vajdy, M., Kosco-Vilbois, M.H., Kopf, M., Kohler, G., and Lycke, N. 1995. Impaired mucosal immune responses in interleukin 4-targeted mice. J. Exp. Med. 181:41-53.

20. Lukacs, N.W., Strieter, R.M., Chensue, S.W., and Kunkel, S.L. 1994. Interleukin-4-dependent pulmonary eosinophil infiltration in a murine model of asthma. Am. J. Respir. Cell Mol. Biol. 10:526-532.

21. Coyle, A.J., et al. 1995. Interleukin-4 is required for the induction of lung Th2 mucosal immunity. Am. J. Respir. Cell Mol. Biol. 13:54-59.

22. Brusselle, G., Kips, J., Joos, G., Bluethmann, H., and Pauwels, R. 1995. Allergen-induced airway inflammation and bronchial responsiveness in wild-type and interleukin-4-deficient mice. Am. J. Respir. Cell Mol. Biol. 12:254-259.

23. Hogan, S.P., Mould, A., Kikutani, H., Ramsay, A.J., and Foster, P.S. 1997. Aeroallergen-induced eosinophilic inflammation, lung damage, and airways hyperreactivity in mice can occur independently of IL-4 and allergen-specific immunoglobulins. J. Clin. Invest. 99:1329-1339.

24. Chensue, S.W., Warmington, K., Ruth, J.H., Lukacs, N., and Kunkel, S.L. 1997. Mycobacterial and schistosomal antigen-elicited granuloma formation in IFN-gamma and IL-4 knockout mice: analysis of local and regional cytokine and chemokine networks [erratum 1999,162:3106]. J. Immunol. 159:3565-3573.

25. von der Weid, T., Kopf, M., Kohler, G., and Langhorne, J. 1994. The immune response to Plasmodium chabaudi malaria in interleukin-4. deficient mice. Eur. J. Immunol. 24:2285-2293.

26. Lawrence, R.A., Gray, C.A., Osborne, J., and Maizels, R.M. 1996. Nippostrongylus brasiliensis: cytokine responses and nematode expulsion in normal and IL-4-deficient mice. Exp. Parasitol. 84:65-73.

27. Barner, M., Mohrs, M., Brombacher, F., and Kopf, M. 1998. Differences between IL-4R alpha-deficient and IL-4-deficient mice reveal a role for IL-13 in the regulation of Th2 responses. Curr. Biol. 8:669-672.

28. McKenzie, G.J., et al. 1998. Impaired development of Th2 cells in IL-13deficient mice. Immunity. 9:423-432

29. Urban, J.F., Jr., et al. 1998. IL-13, IL-4Ralpha, and Stat6 are required for the expulsion of the gastrointestinal nematode parasite Nippostrongylus brasiliensis. Immunity. 8:255-264

30. Donaldson, D.D., et al. 1998. The murine IL-13 receptor alpha 2: molecular cloning, characterization, and comparison with murine IL-13 receptor alpha 1. J. Immunol. 161:2317-2324.

31. Wang, L.F., Lin, J.Y., Hsieh, K.H., and Lin, R.H. 1996. Epicutaneous exposure of protein antigen induces a predominant Th2-like response with high IgE production in mice. J. Immunol. 156:4077-4082.

32. Spergel, J.M., et al. 1998. Epicutaneous sensitization with protein antigen induces localized allergic dermatitis and hyperresponsiveness to methacholine after single exposure to aerosolized antigen in mice. J. Clin. Invest. 101:1614-1622.

33. Temann, U.A., et al. 1997. A novel role for murine IL-4 in vivo: induction of MUC5AC gene expression and mucin hypersecretion. Am. J. Respir. Cell Mol. Biol. 16:471-478.

34. Lee, J.J., et al. 1997. Interleukin-5 expression in the lung epithelium of transgenic mice leads to pulmonary changes pathognomonic of asthma. J. Exp. Med. 185:2143-2156.

35. Cohn, L., Homer, R.J., Marinov, A., Rankin, J., and Bottomly, K. 1997. Induction of airway mucus production by T helper 2 (Th2) cells: a critical role for interleukin 4 in cell recruitment but not mucus production. J. Exp. Med. 186:1737-1747.

36. Hou, J., et al. 1994. An interleukin-4-induced transcription factor: IL-4 Stat. Science. 265:1701-1706.
37. Kaplan, M.H., and Grusby, M.J. 1998. Regulation of T helper cell differentiation by STAT molecules. J. Lenkoc. Biol. 64:2-5.

38. Takeda, K., et al. 1996. Essential role of Stat6 in IL-4 signalling. Nature. 380:627-630.

39. Zurawski, G., and de Vries, J.E. 1994. Interleukin 13, an interleukin 4-like cytokine that acts on monocytes and B cells, but not on T cells. Immunol. Today. 15:19-26.

40. Lin, J.X., et al. 1995. The role of shared receptor motifs and common Stat proteins in the generation of cytokine pleiotropy and redundancy by IL2, IL-4, IL-7, IL-13, and IL-15. Immunity. 2:331-339.

41. Stevens, T.L., et al. 1988. Regulation of antibody isotype secretion by subsets of antigen-specific helper T cells. Nature. 334:255-258.

42. Snapper, C.M., and Paul, W.E. 1987. Interferon-gamma and B cell stimulatory factor-1 reciprocally regulate Ig isotype production. Science. 236:944-947.

43. Holt, P.G., Batty, J.E., and Turner, K.J. 1981. Inhibition of specific IgE responses in mice by pre-exposure to inhaled antigen. Immunology. 42:409-417.

44. McMenamin, C., and Holt, P.G. 1993. The natural immune response to inhaled soluble protein antigens involves major histocompatibility complex (MHC) class I-restricted CD8+ T cell-mediated but MHC class IIrestricted $\mathrm{CD} 4+\mathrm{T}$ cell-dependent immune deviation resulting in selective suppression of immunoglobulin E production. J. Exp. Med. 178:889-899.

45. Renz, H., et al. 1992. Aerosolized antigen exposure without adjuvant causes increased $\operatorname{IgE}$ production and increased airway responsiveness in the mouse. J. Allergy Clin. Immunol. 89:1127-1138.

46. Brusselle, G.G., et al. 1994. Attenuation of allergic airway inflammation in IL-4 deficient mice. Clin. Exp. Allergy. 24:73-80.

47. Spergel, J.M., Mizoguchi, E., Oettgen, H., Bhan, A.K., and Geha, R.S. 1999. Roles of TH1 and TH2 cytokines in a murine model of allergic dermatitis. J. Clin. Invest. 103:1103-1111.

48. Nakajima, H., Sano, H., Nishimura, T., Yoshida, S., and Iwamoto, I. 1994. Role of vascular cell adhesion molecule 1/very late activation antigen 4 and intercellular adhesion molecule $1 /$ lymphocyte function-associated antigen 1 interactions in antigen-induced eosinophil and $T$ cell recruitment into the tissue. J. Exp. Med. 179:1145-1154.

49. Thornhill, M.H., et al. 1991. Tumor necrosis factor combines with IL-4 or IFN-gamma to selectively enhance endothelial cell adhesiveness for $\mathrm{T}$ cells. The contribution of vascular cell adhesion molecule-1-dependent and -independent binding mechanisms. J. Immunol. 146:592-598.

50. Shimoda, K., et al. 1996. Lack of IL-4-induced Th2 response and IgE class switching in mice with disrupted Stat6 gene. Nature. 380:630-633.

51. Akimoto, T., et al. 1998. Abrogation of bronchial eosinophilic inflammation and airway hyperreactivity in signal transducers and activators of transcription (STAT)6-deficient mice. J. Exp. Med. 187:1537-1542.

52. Kaplan, M.H., Schindler, U., Smiley, S.T., and Grusby, M.J. 1996. Stat6 is required for mediating responses to IL-4 and for development of Th2 cells. Immunity. 4:313-319.

53. Zurawski, S.M., et al. 1995. The primary binding subunit of the human interleukin-4 receptor is also a component of the interleukin-13 receptor. J. Biol. Chem. 270:13869-13878.

54. Smerz-Bertling, C., and Duschl, A. 1995. Both interleukin 4 and interleukin 13 induce tyrosine phosphorylation of the $140-\mathrm{kDa}$ subunit of the interleukin 4 receptor. J. Biol. Chem. 270:966-970.

55. Grunig, G., et al. 1998. Requirement for IL-13 independently of IL-4 in experimental asthma. Science. 282:2261-2263.

56. Wills-Karp, M., et al. 1998. Interleukin-13: central mediator of allergic asthma. Science. 282:2258-2261.

57. Doherty, T.M., Kastelein, R., Menon, S., Andrade, S., and Coffman, R.L. 1993. Modulation of murine macrophage function by IL-13. J. Immunol. 151:7151-7160.

58. Yokota, K., et al. 1996. Cytokine-mediated communication between dendritic epidermal T cells and Langerhans cells. In vitro studies using cell lines. J. Immunol. 157:1529-1537. 